\title{
A comparison between ion characteristics observed by the POLAR and DMSP spacecraft in the high-latitude magnetosphere
}

\author{
T. J. Stubbs ${ }^{1,2}$, M. Lockwood ${ }^{3}$, P. Cargill ${ }^{1}$, M. Grande ${ }^{3}$, B. Kellett ${ }^{3}$, and C. Perry ${ }^{3}$ \\ ${ }^{1}$ Space \& Atmospheric Physics, The Blackett Laboratory, Imperial College London, UK \\ ${ }^{2}$ Laboratory for Extraterrestrial Physics, NASA Goddard Space Flight Center, Greenbelt, Maryland, USA \\ ${ }^{3}$ Space Science Department, Rutherford Appleton Laboratory, Oxfordshire, UK
}

Received: 31 October 2002 - Revised: 12 August 2003 - Accepted: 5 September 2003 - Published: 19 March 2004

\begin{abstract}
We study here the injection and transport of ions in the convection-dominated region of the Earth's magnetosphere. The total ion counts from the CAMMICE MICS instrument aboard the POLAR spacecraft are used to generate occurrence probability distributions of magnetospheric ion populations. MICS ion spectra are characterised by both the peak in the differential energy flux, and the average energy of ions striking the detector. The former permits a comparison with the Stubbs et al. (2001) survey of $\mathrm{He}^{2+}$ ions of solar wind origin within the magnetosphere. The latter can address the occurrences of various classifications of precipitating particle fluxes observed in the topside ionosphere by DMSP satellites (Newell and Meng, 1992). The peak energy occurrences are consistent with our earlier work, including the dawn-dusk asymmetry with enhanced occurrences on the dawn flank at low energies, switching to the dusk flank at higher energies. The differences in the ion energies observed in these two studies can be explained by drift orbit effects and acceleration processes at the magnetopause, and in the tail current sheet. Near noon at average ion energies of $\approx 1 \mathrm{keV}$, the cusp and open LLBL occur further poleward here than in the Newell and Meng survey, probably due to convectionrelated time-of-flight effects. An important new result is that the pre-noon bias previously observed in the LLBL is most likely due to the component of this population on closed field lines, formed largely by low energy ions drifting earthward from the tail. There is no evidence here of mass and momentum transfer from the solar wind to the LLBL by nonreconnection coupling. At higher energies $(\approx 2-20 \mathrm{keV})$, we observe ions mapping to the auroral oval and can distinguish between the boundary and central plasma sheets. We show that ions at these energies relate to a transition from dawnward to duskward dominated flow, this is evidence of how ion drift orbits in the tail influence the location and behaviour of the plasma populations in the magnetosphere.
\end{abstract}

Correspondence to: T. J. Stubbs

(tstubbs@lepvax.gsfc.nasa.gov)
Key words. Magnetospheric physics (magnetopause, cusp and boundary layers; magnetosphere-ionosphere interactions; magnetospheric configuration and dynamic)

\section{Introduction}

In order to understand how mass and energy flow into and around the magnetosphere, we must be able to relate plasma populations observed at different locations. This, in turn, requires us to understand the transport mechanisms involved. The coupling between the solar wind/magnetosheath and magnetosphere is driven by magnetic reconnection at the dayside magnetopause, which creates open field lines that allow plasma to cross this boundary. On crossing the magnetopause, magnetosheath plasma is accelerated and then dispersed by convection as it precipitates into the high-latitude dayside magnetosphere creating the open and low-latitude boundary layer (LLBL), as well as the cusp and mantle regions. It has been proposed that other injection mechanisms or re-closure of open field lines by lobe reconnection could produce an LLBL on closed field lines (see review by Lockwood, 1997). The plasma component remaining on these field lines until they are closed by magnetic reconnection in the tail is trapped within the magnetosphere and subsequently accelerated earthward. The velocity of the particles in this component of the plasma increases by the Alfvén speed with each crossing of the midtail current sheet (see Onsager and Mukai, 1995 and references therein). The charged particles in this plasma then follow drift orbits which depend on the convection electric field and the particle charge and energy. Thus, the observed distribution of these particles depends on the above three factors.

A study of the distribution of ions of solar wind origin within the magnetosphere was undertaken by Stubbs et al. (2001) (hereafter referred to as Stubbs et al.), using $\mathrm{He}^{2+}$ ions detected by the CAMMICE MICS instrument aboard 
POLAR as a tracer. In this study each integration period was characterised by the energy at the peak in the differential energy flux $\left(J_{E}\right)$ spectrum between $\approx 1$ and $200 \mathrm{keV} \cdot \mathrm{e}^{-1}$ (hereafter referred to as "the peak energy"), and the occurrence probabilities were plotted as a function of invariant latitude $(\Lambda)$ and magnetic local time (MLT). In the energy range from 1.8 to $21.4 \mathrm{keV}$ an enhancement was observed along the dawn flank between $\Lambda \approx 68^{\circ}$ and $75^{\circ}$, giving rise to a dawn-dusk asymmetry. This asymmetry was shown not to be caused by direct entry of particles into the magnetosphere (i.e. neither the Svalgaard-Mansurov effect nor a dawn-dusk asymmetry in Fermi acceleration at a quasiparallel bow shock), but by ions drifting earthward from the tail, as was demonstrated by sorting the data by energy and the sense of the interplanetary magnetic field $Z$-component (IMF $B_{Z}$ ). The asymmetry was shown to change to the dusk flank at higher energies, with the energy at which the peak in the occurrence flips from the dawn to the dusk flank (hereafter referred to as the "cross-over" energy) being estimated at $\approx 23 \mathrm{keV}$. This flip in the asymmetry occurred at slightly lower energies for northward IMF conditions due to the convection electric field being weaker at these times, a behaviour consistent with that of ions executing drift orbits (Lyons and Williams, 1984b). The cusp was isolated in this study at energies between 1.8 and $6.9 \mathrm{keV}$.

The precipitation into the dayside ionosphere at low altitudes was classified and mapped by Newell and Meng (1992) (hereafter referred to as Newell and Meng) from observations made by the SSJ/4 spectrometers aboard the Defense Meteorological Satellite Program (DMSP) spacecraft. They used an automated identification scheme to produce occurrence frequency maps for observing various types of plasma precipitating into the ionosphere. The classifications and results from this survey are discussed in more detail in Sect. 4. The fleet of DMSP spacecraft are in Sun synchronous polar orbits at an altitude of around $830 \mathrm{~km}$. The SSJ/4 instruments are particle spectrometers which measure precipitating electron and ion fluxes between $30 \mathrm{eV}$ and $30 \mathrm{keV}$ every second (Hardy et al., 1984). The look direction is within a few degrees of the local vertical, in order to detect precipitating particles with pitch angles inside the loss cone. Stubbs et al. carried out a brief comparison between their $\mathrm{He}^{2+}$ ion occurrences and the Newell and Meng survey and showed them to be qualitatively consistent.

More recently, Friedel et al. (2001) studied the access of plasma sheet particles into the inner magnetosphere using data from the Hydra hot plasma experiment (Scudder et al., 1995) aboard POLAR. Using a formulation introduced by Whipple (1978), they showed that, in general, the occurrence distributions of ions and electrons were globally consistent with the conventional drift paradigm; thus, validating the simple corotation and convection electric fields used in their analysis. They were also able to describe the average particle transport over a wide range of geomagnetic activity levels. This result was based mainly on the electron data which was very well ordered by the Alfvén boundaries (see Wolf, 1995). The ion data, however, was not that well or- ganised, probably due in part to the more complex ion drift orbits.

There is an overlap in the range of energy detection of the $\mathrm{SSJ} / 4$ spectrometers and MICS from $\approx 1$ to $30 \mathrm{keV}$. In order to compare our results with the Newell and Meng survey, and other instruments that measure total ion flux, such as POLAR/Hydra, we survey the total ion signature at POLAR using data from the MICS DCR (Double Coincidence Rate) channel. In characterising the different types of particle precipitation, Newell and Meng use the average energy of ion precipitation striking the atmosphere as a criterion to distinguish between precipitation types. Therefore, we survey the total ion flux at POLAR, using both the peak and average ion energies as a bridge between the Stubbs et al. survey and the work of Newell and Meng. Our aim is to understand how the ion injection and transport processes invoked to explain the observations of Stubbs et al. and Friedel et al. (2001) relate to the precipitating particle occurrence frequency distributions observed in the ionosphere by Newell and Meng. In Sect. 2 we give a brief description of the POLAR spacecraft and the CAMMICE MICS instrument used in this study. Sect. 3 gives details of the data processing and presentation used in this work, and includes a discussion of particle fluxes and coordinate systems. The various dayside particle precipitation characteristics used by Newell and Meng are described in Sect. 4, and are important for relating their results with those presented in this paper. Sect. 5 contains the results and interpretation: Sect. 5.1 concentrates on the peak ion energy results and the comparison with the work of Stubbs et al.; Sect. 5.2 discusses the average ion energy results and focuses more on the comparison with the Newell and Meng survey. Finally, in Sect. 6, we present the discussion and conclusions.

\section{Spacecraft and instrumentation}

The POLAR spacecraft is in a $1.8 \times 9 R_{E}$ polar orbit with an inclination of $\approx 86^{\circ}$ to the equator and a period of $\approx 17.5 \mathrm{~h}$ (Acuña et al., 1995). Its apogee is at high northern latitudes and is spin-stabilised at $10 \mathrm{rpm}$, with its spin axis approximately perpendicular to the orbital plane. The data set used in this study is from the Magnetospheric Ion Composition Sensor (MICS) of the Charge and Mass Magnetospheric Ion Composition Experiment (CAMMICE). The Electrostatic Analyser (ESA) on MICS sorts by energy per charge (E/q) and has 32 steps (0-31), with E/q mid-points ranging from 1.0-416.7 keV.e $\mathrm{e}^{-1}$. The ESA has an energy resolution $\Delta \mathrm{E} / \mathrm{E} \approx 16 \%$ full width at half maximum, so the $\mathrm{E} / \mathrm{q}$ range of the detector is effectively from 0.9 to $450.0 \mathrm{keV} \cdot \mathrm{e}^{-1}$. For most of the mission, only steps 0-24 have been used, reducing the effective E/q range to $0.9-208.8 \mathrm{keV}_{\mathrm{e}} \mathrm{e}^{-1}$. Sorting by the ESA, combined with time-of-flight measurements and determination of the energy deposited in the solid state detector, allows the MICS to determine the flux, mass and charge states of the incident ions (Wilken et al., 1992; Fritz et al., 1997). MICS has a temporal resolution of $197.5 \mathrm{~s}$; it 
takes data at one ESA step per rotation (6s), thereby requiring $192 \mathrm{~s}$ to make an observation at every ESA step, plus $5.5 \mathrm{~s}$ to reset for the next set of measurements. Raw count rates (counts. $\mathrm{s}^{-1}$ ) are calculated by averaging over the spacecraft spin period $(6 \mathrm{~s})$.

\section{Data processing and presentation}

We survey the DCR ion energies in the E/q mid-point range of $1.0-23.3 \mathrm{keV} \cdot \mathrm{e}^{-1}$. The DCR counts are dominated by protons with a charge state of +1 , for which this is equivalent to an energy range of $1.0-23.3 \mathrm{keV}$. For studies of the peak energy this gives an effective energy range of $0.9-25.2 \mathrm{keV}$ due to the spread of energies about the channel midpoints given by the energy resolution. As was done in Stubbs et al., we search for the peak in the $J_{E}$ spectra over the whole energy range $\left(\approx 1-200 \mathrm{keV} \cdot \mathrm{e}^{-1}\right)$. To calculate the average ion energy we use ESA channels from 0 to 12, corresponding to midpoints from 1.0 to $30.9 \mathrm{keV} . \mathrm{e}^{-1}$; this is done to achieve the best possible comparison with average ion energies determined from the SSJ/4 instruments aboard DMSP. For the average energy studies we bin the data using the same energy midpoints as the E/q channels. However, the average energy for each bin extends to the midpoint between each E/q channel, this is calculated using logarithmic interpolation. Therefore, the average ion energies in the survey range from 0.9 to $26.83 \mathrm{keV}$.

To avoid using single counts, which can be created by anomalous events in the detectors, we set the minimum raw count rate at 1 count. ${ }^{-1}$, so 6 counts in an integration period are required for it to register ions as present. A maximum raw count rate of 2500 counts. $^{-1}$ was also set, as cosmic rays striking a detector can lead to anomalously high count rates $\left(\approx 10000\right.$ counts. $\mathrm{s}^{-1}$ in the DCR channel).

We calculate the average energy, $\langle E\rangle$, from the ratio of the integral energy flux $\left(F_{E}\right)$ to the integral number flux $(F)$ :

$$
<E>=\frac{F_{E}}{F}=\frac{\sum_{i=L}^{U} J_{E_{i}}}{\sum_{i=L}^{U} J_{i}}=\frac{\sum_{i=L}^{U} C_{C i}}{\sum_{i=L}^{U} \frac{C_{C i}}{E_{i}}} .
$$

In the above equation: $J_{E i}$ and $J_{i}$ are the differential energy and differential number fluxes per channel, respectively; $L$ and $U$ represent the lower and upper ESA steps, respectively, in this case $L=0$ and $U=12$ (energy midpoint of $\left.30.9 \mathrm{keV} . \mathrm{e}^{-1}\right) ; C_{C i}$ and $E_{i}$ are the corrected count rate and energy detected at each channel, respectively. $\left(C_{C}\right.$ is determined by dividing the raw count rate by the channel efficiency.) As the geometric factor is the same for all MICS channels, it can be removed from the differential flux per channel terms and cancelled in Eq. (1); thus, leaving just the $C_{C i}$ and $E_{i}$ terms in the equation (for a more detailed discussion of particle instrument fluxes, see Stubbs, 2002). This eliminates the need for absolute fluxes, as is the case for determining the peak energy.
The method for calculating average ion energy shown in Eq. (1) is used by the Air Force Research Laboratory (AFRL) when analysing DMSP SSJ/4 particle data (Newell, personal communication). This definition of $\langle E\rangle$ corresponds to the average energy of particle precipitation striking the atmosphere or the detector. (This does not correspond to the average energy moment of the distribution function.) The $<E>$ value given by Eq. (1) is weighted toward the higher energy particles, as more of the higher energy faster moving particles strike the detector/atmosphere per unit time than the slower low energy particles. As a result, it has a higher value than the average energy given by the moment of the distribution function, which considers the average energy of particles in a given volume of phase space.

In order to facilitate a direct comparison with Stubbs et al., we plot the occurrence probability of observing ions based on the peak energy selection criteria as a function of invariant latitude $(\Lambda)$ and magnetic local time (MLT), as shown in Fig. 2. We calculate invariant latitude using the McIlwain $L$ parameter, also referred to as $L_{m}$ or $L$-shell (Mcllwain, 1966). This is calculated using the SHELLIG routines (available from NSSDC FTP site), which uses the IGRF magnetic field model. As we are interested in particles on drift orbits, where the magnetic moment and the second adiabatic invariant are generally conserved, these coordinates should order the data well.

The occurrences based on the average energy selection criteria, shown in Fig. 3, are plotted as a function of Corrected Geomagnetic (CGM) latitude and MLT. The CGM coordinates, as described by Baker and Wing (1989), were used by Newell and Meng to order their data. (CGM latitude is later referred to as MLAT, to be consistent with the nomenclature used by Newell and Meng.) As the code for calculating CGM coordinates (available from the JHU/APL website) is based on the IGRF model and limited to altitudes below $40000 \mathrm{~km}$, we traced field lines from POLAR to the Earth's surface using the T01 model (Tsyganenko, (2002a, b) before converting into CGM coordinates. T01 is an empirical model that has been generated using a large database of magnetic field observations. This has included data from the Magnetic Field Experiment (MFE) aboard POLAR, which has improved the modelling of the dayside Birkeland current systems. As near field-aligned particles tend to follow magnetic field lines, this should relate our study well to the occurrence frequencies of Newell and Meng. Assuming that there are no systematic errors in the T01 field line mapping, we would expect differences to be due to energy and pitch angle dependent dispersion caused by the convection of field lines during the time-of-flight from the reconnection $X$-line to POLAR and DMSP altitudes. The T01 model is dependent on the solar wind dynamic pressure, IMF $B_{Y}$ and $B_{Z}$, and the $D_{s t}$ index, as well as time and location. These solar wind parameters were obtained from the Magnetic Field Investigation (MFI) and Solar Wind Experiment (SWE) aboard the WIND spacecraft. The MFI and SWE datasets were validated, joined and time lagged to the Earth, using the techniques described in Stubbs et al. (2003). 

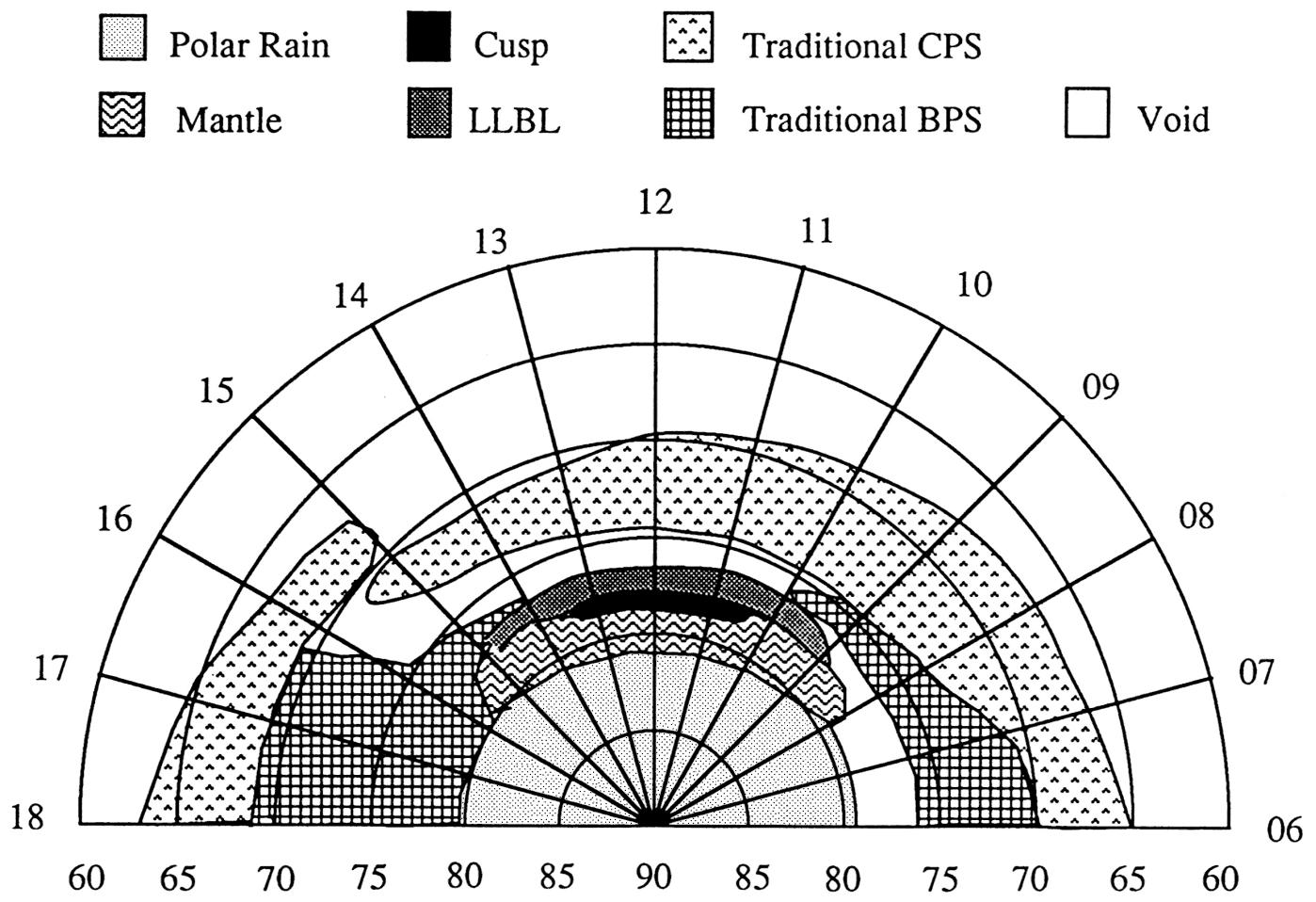

Fig. 1. A map of the ionosphere to the magnetosphere based on plasma characteristics as a function of MLAT and MLT (adapted from Newell and Meng, 1992). Data was taken for all interplanetary conditions (@ by American Geophysical Union).

The MLT in both cases is calculated using the method described by Baker and Wing (1989), which takes into account effects due to seasonal changes and the eccentricity of the Earth's orbit. Though, of course, it is calculated using the magnetic longitude at the spacecraft for the peak ion survey (Fig. 2) and the CGM longitude at the T01 footpoint for the average ion energy survey (Fig. 3).

The differences between $\Lambda$ and MLAT (calculated in the manner described above) are most noticeable at highlatitudes, where $\Lambda$ tends to place observations further poleward than MLAT. This is because, unlike the T01 model, the near-dipolar IGRF model used in determining $\Lambda$ takes no account of the distortion of the magnetospheric field from external currents. This distortion is primarily due to the compression of the magnetosphere, particularly at the dayside magnetopause. This means that magnetospheric field lines are moved poleward relative to the more dipolar IGRF field lines, hence, the discrepancy between $\Lambda$ and MLAT. Also, when calculating MLAT we follow a magnetic field line to the Earth's surface along a magnetic shell; whereas in calculating $\Lambda$ we trace along an $L$-shell where adiabatic invariants are conserved. This also leads to a further discrepancy between $\Lambda$ and MLAT; however, compared to the differences due to external currents this is negligible.

The occurrence plots in Figs. 2 and 3 cover latitudes from $60^{\circ}$ to $90^{\circ}$ at all MLTs in $1.5^{\circ}$ by 24 min bins. (The survey was also carried out for smaller bins of $0.5^{\circ}$ width in latitude and all the features reported here were evident, with the addition of a slightly increased statistical fluctuation level be- cause sample numbers in each bin were lower.) The occurrence probability was calculated by dividing the number of times POLAR observed ions by the total number of samples made in that bin. Plotting occurrence probabilities removes any biasing in the data caused by the orbit or instrument sampling. To ensure all bins are statistically significant, we have removed any bins sampled less than 50 times. This means an anomalous event will only register a $2 \%$ probability at most. The occurrence probability scale bar was standardised from 0 to $70 \%$ in the plots, to allow for a direct comparison. The data coverage in this study has been extended from that used in Stubbs et al. to be almost continuous from 18 March 1996 to 31 March 2000.

An important consideration when comparing data from the DMSP SSJ/4 instruments and MICS is that the SSJ/4 observe only precipitating ions in the loss cone which would have been near field-aligned at the greater altitude of the POLAR satellite, whereas MICS observes both precipitating magnetospheric and outflowing ionospheric ion populations at a range of pitch angles. One such source of ionospheric plasma is the polar wind. In the region of the cusp, the cleft ion fountain also populates the magnetosphere with $\mathrm{O}^{+}$and $\mathrm{H}^{+}$ions, particularly during active times (Shelley, 1986; Lockwood et al., 1985; Hultqvist et al., 1988). Ionospheric outflow has been observed in the auroral regions in the form of ion beams and conics with energies of 0.1 to $10 \mathrm{keV}$ (e.g. Mizera and Fennell, 1977; Ghielmetti et al., 1978). This could possibly lead to significant discrepancies between our survey and that of Newell and Meng. 

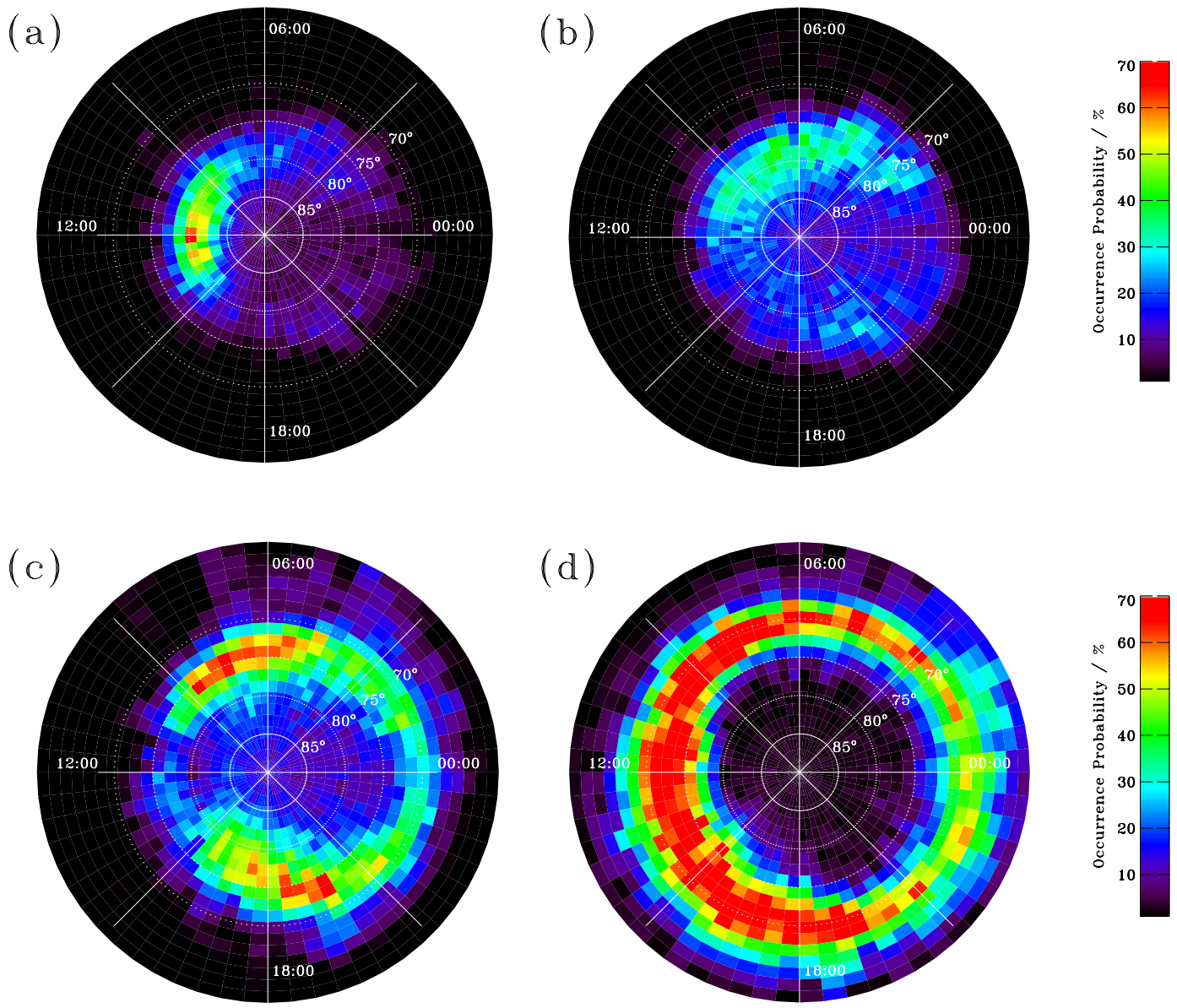

Fig. 2. The occurrence probability of ions in the magnetosphere plotted as invariant latitude $(\Lambda)$ versus magnetic local time (MLT) in polar coordinates. Occurrence probabilities for energy ranges: (a) $0.9-1.1 \mathrm{keV}$, (b) $1.2-2.6 \mathrm{keV}$, (c) $2.9-8.0 \mathrm{keV}$ and (d) $9.1-25.2 \mathrm{keV}$. The peak in the DCR (total ion signature) differential energy flux spectrum $\left(J_{E}\right)$ is used to determine a characteristic energy for each integration period. $\Lambda$ ranges from $60^{\circ}$ to $90^{\circ}$ for all MLTs with a bin size of $1.5^{\circ}$ by $24 \mathrm{~min}$. Each bin has a 50-sample threshold to ensure reliable statistics. Data has been taken for all interplanetary conditions. CAMMICE data used in this plot was taken almost continuously from 18 March 1996 to 31 March 2000.

\section{The dayside particle precipitation classifications used by Newell and Meng}

The identification of the various types of particle precipitation by Newell and Meng was done using a neural network technique described by Newell et al. (1991b). This technique was adopted by these authors as it can be difficult to distinguish quantitatively between different types of precipitation, although conceptually there is often a clear distinction.

In Table 1 we list the particle precipitation classifications that are most relevant to our study, with typical average ion energy values and integral fluxes based on descriptions and examples given in Newell et al. (1991b, c). It is important to note that the classification of particle precipitation summarised here is based on both electron and ion spectra; therefore regions with similar ion characteristics (e.g. void and polar rain) are distinguished by their electron spectra. The schematic in Fig. 1 shows a map of ionospheric precipitation based on the results from Newell and Meng. It is important to note that in the following discussions we compare our results with the original Newell and Meng occurrence frequency plots shown in their Fig. 1, and not the schematic shown in their Fig. 2 (and reproduced here in Fig. 1). In their survey, the absence of any significant precipitation is termed void $\left(\approx 0.01 \mathrm{ergs} . \mathrm{cm}^{-2} \cdot \mathrm{s}^{-1}\right)$. Polar rain is typically observed as low energy electron precipitation in the polar cap often during southward IMF, as identified by Winningham and Heikkila (1974). Mantle is classed as a region of low energy ion precipitation poleward of the dayside auroral oval, as described by Newell et al. (1991a). Cusp refers to magnetosheath plasma that has gained direct entry into the magnetosphere. Cusp ions are characterised as having low temperature with high bulk flow velocity; this is described at length in Newell and Meng (1988, 1990). Plasma in the low latitude boundary layer (LLBL) is described as "varying considerably", but generally exhibits higher thermal temperatures and lower bulk flow than in the magnetosheath, and contains high energy particles of magnetospheric origin near the magnetopause, as described by Eastman et al. (1985) and Sckopke et al. (1981). 

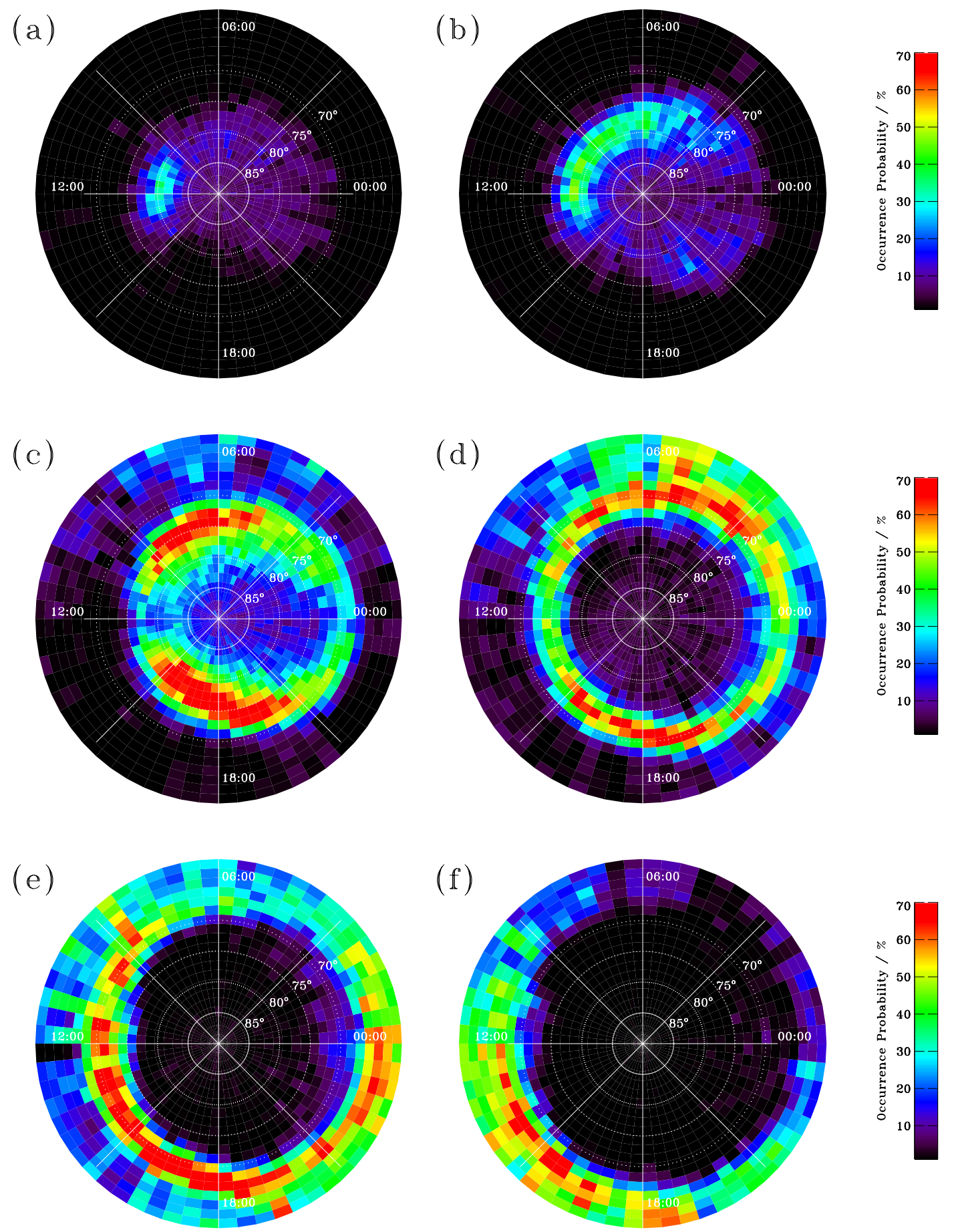

Fig. 3. The occurrence probability of average ion energies: (a) $0.9-1.53 \mathrm{keV}$, (b) $1.53-2.10 \mathrm{keV}$, (c) $2.10-4.85 \mathrm{keV}$, (d) $4.85-8.55 \mathrm{keV}$, (e) $8.55-15.20 \mathrm{keV}$ and (f) $15.20-26.83 \mathrm{keV}$. In each integration period, the average energy of ions between 0.9 and $30.9 \mathrm{keVe}$ detected by the DCR channel is determined. (Average ion energy $\left\langle E>=F_{E} / F\right.$.) These occurrences are plotted as a function of MLAT and MLT of the footpoints of T01 model field lines mapped from POLAR. Otherwise, data is presented in the same format as Fig. 2.

The boundary plasma sheet (BPS) and central plasma sheet (CPS) are the regions where the discrete and diffuse aurorae are observed, respectively, as described by Winningham et al. (1975). The defining characteristic of CPS precipitation is given to be high-energy plasma (several keV electrons and tens of keV ions) with comparatively little spatial or spectral structure. The BPS lies poleward of the CPS and is characterised by slightly lower energies and intensities and more spatially and spectrally structured ion and electron spectra than observed in the CPS. However, the key to distinguishing BPS from CPS precipitation is the fact that most discrete auroral arcs and acceleration events are embedded 
Table 1. Typical average ion energies and integral fluxes used as part of the ionospheric precipitation classification (based on examples given in Newell et al. 1991b, c). Acronyms used: LLBL - Low-latitude Boundary Layer, BPS - Boundary Plasma Sheet, CPS - Central Plasma Sheet. These regions are described in Sect. 4.

\begin{tabular}{lcc}
$\begin{array}{l}\text { Classification of } \\
\text { Particle Precipitation }\end{array}$ & $\begin{array}{c}\text { Average Ion Energy/ } \\
\mathrm{eV}\end{array}$ & $\begin{array}{c}\text { Integral Energy Flux/ } \\
10^{8} \mathrm{eV} \cdot \mathrm{cm}^{-2} \cdot \mathrm{s}^{-1}\end{array}$ \\
\hline Void & - & $1.0-3.0$ \\
Polar Rain & - & $1.0-3.0$ \\
Mantle & $300-1000$ & $1.0-100$ \\
Cusp & $800-2000$ & $300-1000$ \\
LLBL & $3000-5000$ & $100-500$ \\
BPS (Midnight/Dusk) & $10000-25000$ & $10-100$ \\
BPS (Noon/Dawn) & $1000-10000$ & $2.0-300$ \\
CPS (Midnight/Dusk) & $10000-30000$ & $30-300$ \\
CPS (Noon/Dawn) & $300-8000$ & $1.0-50$ \\
\hline
\end{tabular}

within the lower energy background BPS electron precipitation. Feldstein and Galperin (1985) and Galperin and Feldstein (1991) argue that the CPS maps to the near-Earth region of quasi-dipolar field lines; and the BPS to the distant plasma sheet and to the plasma sheet boundary layer (PSBL). However, as all PSBL field lines cross the tail neutral sheet, the CPS/PSBL distinction is to some extent qualitative (Newell et al., 1991c). From this description, and the fact that ion energies are greater around dusk and midnight than dawn and noon (see Table 1), we would expect the CPS and BPS regions to relate to the dawn-dusk asymmetry discussed by Stubbs et al.

\section{Results and interpretation}

5.1 DCR peak ion energy occurrence distributions and comparison with Stubbs et al. $\mathrm{He}^{2+}$ ion survey

At the ion energies detectable by MICS in the cusp region we are observing the high-energy tail of the cusp ion distribution. Under southward IMF conditions, the cusp particles are accelerated at the dayside magnetopause while crossing the rotational discontinuity (RD) resulting from subsolar reconnection. Under northward IMF conditions, particles are also accelerated on crossing an $\mathrm{RD}$, if the magnetosheath flow adjacent to the tail-lobe is sub-Alfvénic such that reconnection can occur. By analysing a time stationary RD in the de Hoffman-Teller (dHT) frame and then transforming back into the Earth's frame we obtain the Walén relation

$\boldsymbol{V}=\boldsymbol{V}_{H T} \pm \boldsymbol{V}_{A}$

(Hudson, 1970). In this equation $\boldsymbol{V}$ is the plasma velocity; $V_{H T}$ is the speed of the dHT frame (in which the electric field is zero) and can be thought of as the velocity of field line motion along the magnetopause; and $\boldsymbol{V}_{A}$ is the local Alfvén speed $\left(\boldsymbol{V}_{A}=\boldsymbol{B} /\left(\mu_{0} \rho\right)^{1 / 2}\right)$. The + and - apply on opposite sides of the reconnection $X$-line and depend on the sense of the boundary-normal field. Depending on the orientation of
$\boldsymbol{V}_{H T}$ with respect to $\boldsymbol{B}$ (and hence $\boldsymbol{V}_{A}$ ), plasma is either accelerated or decelerated by $\boldsymbol{V}_{A}$ on crossing an RD. Therefore, the associated increase in particle energy is proportional to the particle mass, as all particles receive the same change in velocity on crossing the RD.

Clearly the relative increase in energy received by $\mathrm{H}^{+}$and $\mathrm{He}^{2+}$ ions is dependent on their initial energies in the magnetosheath and $\boldsymbol{V}_{A}$. In the depletion layer around the subsolar magnetosheath, proton temperatures are typically about $3.8 \times 10^{6} \mathrm{~K}$ and plasma flow is of the order of $100 \mathrm{~km} . \mathrm{s}^{-1}$ under high magnetic shear conditions (Phan et al., 1994). For low magnetic shear conditions, temperatures in the dataset presented by these authors were slightly lower and plasma flows were a little faster. $\boldsymbol{V}_{A}$ at the dayside magnetopause varies between around 100 and $300 \mathrm{~km} \cdot \mathrm{s}^{-1}$, but is typically about $200 \mathrm{~km} . \mathrm{s}^{-1}$ (Phan et al., 1996). Fuselier et al. (1991) showed that in the depletion layer the ratio of $\mathrm{He}^{2+}$ to $\mathrm{H}^{+}$ temperatures is typically $\approx 4.5$. From these assumptions, we estimate that $\mathrm{He}^{2+}$ and $\mathrm{H}^{+}$ions in the depletion layer have energies of around $2.6 \mathrm{keV}$ and $0.6 \mathrm{keV}$, respectively. In the cusp, subsequent to being accelerated across the RD, we would expect $\mathrm{He}^{2+}$ and $\mathrm{H}^{+}$ion energies of around $3.5 \mathrm{keV}$ and $0.8 \mathrm{keV}$, respectively, a difference of about a factor of 4 . For tail-lobe reconnection we would expect a similar difference between $\mathrm{He}^{2+}$ and $\mathrm{H}^{+}$ion energies in the cusp. Although the initial temperatures in the magnetosheath would be lower and the acceleration at the RD would be significantly less, the bulk flow in the magnetosheath, however, would be considerably faster.

For particles drifting in from the tail it is harder to predict their energies, as they have more complicated trajectories. The $\boldsymbol{E} \times \boldsymbol{B}$ drift acts towards the dawn flank and is dependent only on the ambient electric and magnetic fields. The gradient/curvature drift acts towards the dusk flank and depends on particle energy and charge. Using a crude analytical analysis we can estimate the energy at which these two competing drifts balance, referred to here as the cross-over energy. If we consider the dipole field case, we can determine the average velocities $\left\langle v_{E}\right\rangle$ and $\left\langle v_{B}\right\rangle$ for the $\boldsymbol{E} \times \boldsymbol{B}$ 


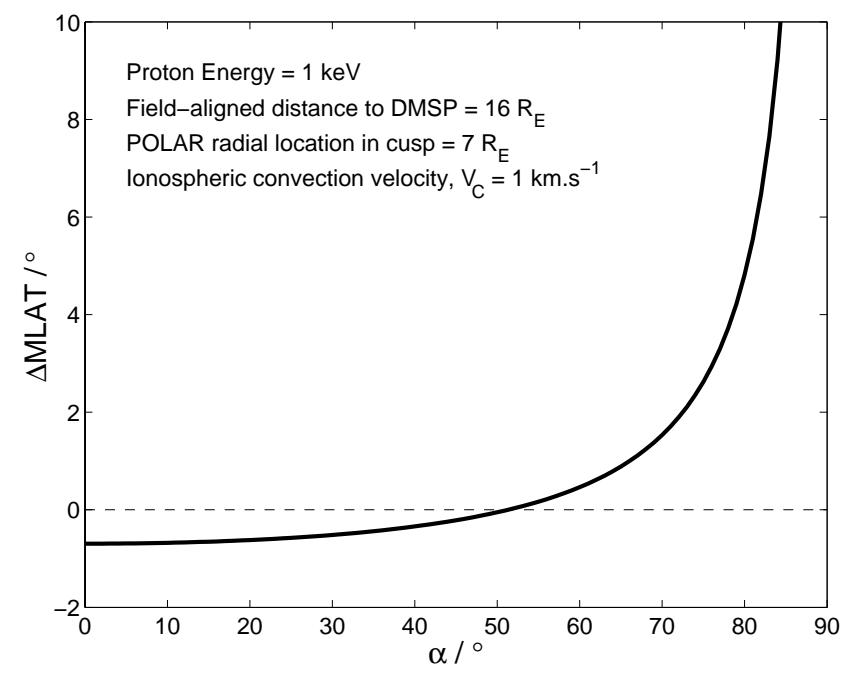

Fig. 4. The estimated difference in the MLAT of $1 \mathrm{keV} \mathrm{H}^{+}$cusp ions observed at POLAR and DMSP $(\triangle \mathrm{MLAT})$ as a function of ion pitch angle $(\alpha) . \triangle$ MLAT $>0$ indicates that cusp ions are observed further poleward at POLAR than at DMSP, and vice versa. Here we have assumed a field-aligned distance from the subsolar reconnection X-line to DMSP and POLAR of $\approx 15 R_{E}$ and $\approx 9 R_{E}$, respectively; and an ionospheric convection velocity, $V_{C}=1 \mathrm{~km} \cdot \mathrm{s}^{-1}$.

and gradient/curvature drift motions, respectively, by integrating over the gyration and bounce motions. If the plasma distribution function is isotropic, then the energy at which $\left\langle v_{E}>/\left\langle v_{B}\right\rangle=1\right.$, i.e. the cross-over energy, is given by

$K_{c e} \approx G Q E_{C} L=2.3 Q E_{C} L$

in units of $\mathrm{keV}$ (see Appendix $\mathrm{A}$ for a derivation of this equation). In the above equation: $Q$ is the charge state (dimensionless integer); $E_{C}$ is the convection electric field $\left(\mathrm{mV} . \mathrm{m}^{-1}\right) ; L$ is the magnetic shell (dimensionless); $G$ is a constant $\left(\mathrm{keV} .\left(\mathrm{mV} \cdot \mathrm{m}^{-1}\right)^{-1} \cdot R_{E}{ }^{-1}\right)$. This equation tells us that for the same ambient conditions, the cross-over energy is proportional to charge. In addition to this drift effect, $\mathrm{He}^{2+}$ ions are also accelerated to higher energies relative to $\mathrm{H}^{+}$ ions during each crossing of the mid-tail current sheet (Onsager and Mukai, 1995). (This is the same process that occurs at the magnetopause rotational discontinuity discussed above.) As a result, we expect a dawn-dusk asymmetry in the $\mathrm{H}^{+}$ions that flips at energies at least a factor of two less than observed in the Stubbs et al. $\mathrm{He}^{2+}$ ion survey. Given that $K_{c e} \approx 23 \mathrm{keV}$ for $\mathrm{He}^{2+}$ ions, we would, therefore, expect the cross-over energy to be below $\approx 10 \mathrm{keV}$ for $\mathrm{H}^{+}$ions.

Figure 2a shows that for peak ion energies from 0.9 to $1.1 \mathrm{keV}$, the occurrences are concentrated around noon, with a slight bias toward the pre-noon sector, and centred at $\Lambda \approx 81^{\circ}$. This corresponds to the region previously identified as the cusp in Figs. $6 \mathrm{a}$ and $6 \mathrm{~b}$ of the Stubbs et al. We also note that the peak ion energies in the cusp $(\approx 1 \mathrm{keV})$ are around a factor of four less than those observed in Stubbs et al. $(\approx 4.3 \mathrm{keV})$, broadly consistent with higher $\mathrm{He}^{2+}$ temperatures in the depletion layer and the effects of acceler- ation across a rotational discontinuity at the magnetopause discussed above. Discrepancies between predicted and observed ion energies are likely due to the fact that a large portion of the cusp ion distribution is at energies below $1 \mathrm{keV}$, as well as the errors involved in this type of calculation for statistical observations based on typical values. This distribution extends further around the dawn flank, suggesting that we are also observing part of the LLBL identified in Fig. 1d of Newell and Meng (on either open or closed field lines). Included in these occurrences are also intervals when POLAR traversed the magnetosheath, as discussed in the Stubbs et al. (2003) survey of extended cusp-like regions observed by CAMMICE MICS. In terms of the peak ion energy used in this study, these occurrences are impossible to distinguish from those on open field lines within the magnetosphere.

We also sorted the cusp occurrences shown in Fig. 2a by the sense of the IMF $B_{Z}$ component (not shown), which showed that the cusp occurred at these energies under both northward and southward IMF conditions. The occurrences around noon for southward IMF conditions were higher than for northward IMF conditions, so we would expect the southward IMF cusp to be more dominant in Fig. 2a. As expected, the southward IMF cusp was observed further equatorward. The cusp had a pre-noon bias for both senses of IMF $B_{Z}$, with the bias being strongest for northward IMF. The southward IMF cusp was broader in $\Lambda\left(\approx 6^{\circ}\right.$ compared with $\left.\approx 4.5^{\circ}\right)$ and narrower in MLT $(\approx 3 \mathrm{~h}$ compared with $\approx 4 \mathrm{~h}$ ). This is consistent with the arguments presented above. Figure $2 \mathrm{~b}$ shows the occurrences for energies from 1.2 to $2.6 \mathrm{keV}$, which clearly isolate the asymmetric dawn feature centred around $\Lambda \approx 79^{\circ}$, as previously shown in Stubbs et al. (Figs. 6c and 6d). Due to the higher sampling rates afforded by the DCR channel, this asymmetry is more distinct than in the data from the $\mathrm{He}^{2+}$ ion channel. From comparison with the LLBL and BPS occurrences shown in Figs. 1d and 1e, respectively, of Newell and Meng, the occurrences here appear to have contributions from the LLBL in the pre-noon sector and the BPS further dawnward around 06:00 MLT. Together with the pre-noon bias in the occurrences observed in Fig. 2a, it appears that a large component of the LLBL observed here in the pre-noon sector is on closed field lines and has been formed from low energy ions convecting earthward from the tail. Also, any LLBL ions in Fig. 2a appear closer to noon than those observed in Fig. 2b. This is consistent with drift motion theory (Lyons and Williams, 1984b), which predicts that lower energy ions will drift around to the dayside as opposed to being scattered at the morning side of the magnetopause. The formation of the LLBL, and whether or not it is on open or closed field lines, has important implications for the nature of the coupling between the solar wind and the magnetosphere (e.g. Lockwood, 1997).

For peak ion energies ranging from 2.9 to $8.0 \mathrm{keV}$ the occurrences are roughly symmetric about the noon-midnight meridian, as shown by Fig. 2c. This suggests that we have isolated the range of energies where the opposing $\boldsymbol{E} \times \boldsymbol{B}$ and gradient/curvature drifts balance: the crossover energy. This appears to correspond to a transition between the ions 
observed in Stubbs et al. (Figs. 6c and 6d, Figs. 6e and 6f). We also notice that the occurrences are more poleward on the dusk flank (centred about $\Lambda \approx 77^{\circ}$ ) than on the dawn flank (centred about $\Lambda \approx 75^{\circ}$ ); this looks similar to the BPS distribution presented in Fig. 1e of Newell and Meng. This difference in the $\Lambda$ of peak occurrence on the dawn and dusk flanks indicates that the drift orbits take ions closer to the Earth on the dawn flank at these energies, and appears to be consistent with the drift orbits shown in Lyons and Williams (1984b) at the transition between dawn and dusk dominated flow.

At the higher energies ranging from 9.1 to $25.2 \mathrm{keV}$ the asymmetry has made the change from the dawn to the dusk flank, with the highest occurrences around the noon and postnoon sectors, as shown in Fig. 2d. This appears to relate to the distributions shown in Figs. 6e and $6 \mathrm{f}$ of Stubbs et al., where we also observe occurrences ranging from $\Lambda \approx 66^{\circ}$ to $76^{\circ}$ and extending over a large range of local times. This is again consistent with ion drift trajectories predicted at these energies where ions drift around to the dayside from dusk and scatter at the magnetopause before reaching the dawn flank (Lyons and Williams, 1984b). These occurrence probabilities appear to relate in part to the CPS population identified in Fig. If of Newell and Meng.

From the observations in Figs. $2 \mathrm{c}$ and $2 \mathrm{~d}$ the cross-over energy appears to be at about $\approx 9 \mathrm{keV}$. As predicted, this is over a factor of two less than the cross-over energy observed for $\mathrm{He}^{2+}$ ions by Stubbs et al. $\left(K_{c e} \approx 23 \mathrm{keV}\right)$. Therefore, this result is consistent with drift orbits and ion acceleration in the mid-tail current sheet, as discussed earlier.

5.2 DCR average ion energy occurrence distributions and comparison with Newell and Meng DMSP survey

In this section, we concentrate on comparing the distribution of the DCR average ion energy occurrence probabilities with the study by Newell and Meng. Figure 3a shows the DCR average ion energies from 0.9 to $1.53 \mathrm{keV}$. In this figure we can identify the cusp around noon, centred at MLAT $\approx 80.5^{\circ}$ and $\approx 11: 45$ MLT, with an MLT extent of $\approx 3$ h noticeably biased toward the pre-noon sector. The occurrence of cusp in Newell and Meng's survey, as shown in their Fig. 1b, is confined - extending only $2.5 \mathrm{~h}$ in MLT and centred at MLAT $\approx 78.5^{\circ}$ and $\approx 11: 45$ MLT. Both extend for $\approx 5^{\circ}$ in MLAT.

The discrepancy in cusp latitudes of around $2^{\circ}$ in MLAT between observations made at POLAR and DMSP is consistent with the result of convection effects. The precipitating ions observed at low altitudes by DMSP are highly field-aligned when they leave the magnetopause: pitch angles increase up to near $90^{\circ}$ only close to the satellite, where the field strength is very high. The instruments on DMSP satellites do not resolve pitch angle and in the cusp region, where elapsed times since reconnection are relatively small, observed fluxes are dominated by ions that have the shortest time-of-flight from the reconnection site to the ionosphere, i.e. field-aligned ions. (This is especially true at energies above $1 \mathrm{keV}$, as used in the survey presented here.) At POLAR altitudes, MICS observes a much broader range of pitch angles and as a result the time-of-flight of the ions varies considerably for any given energy observed. In the cusp, MICS tends to observe ion pitch angle distributions peaking around the field-perpendicular directions at pitch angles of $90^{\circ}$ and $270^{\circ}$, as well as often missing the fieldaligned and anti-field-aligned populations (at pitch angles of $0^{\circ}$ and $180^{\circ}$, respectively); this is due to the magnetic field direction moving out of the plane observed by the instrument, which is perpendicular to the satellite spin axis (e.g. Stubbs et al., 2000).

Figure 4 shows the estimated differences in MLAT as a function of ion pitch angle $(\alpha)$ expected for $1 \mathrm{keV}$ cusp ions. Here we have assumed a typical field-aligned distance of $\approx 15 R_{E}$ from the reconnection $X$-line to the ionosphere; and that POLAR is typically at a radial distance of $\approx 7 R_{E}$ when observing the cusp (i.e. we assume a $\approx 9 R_{E}$ field-aligned distance from POLAR to the $X$-line). The T01 model is used to determine the field-aligned distances used to compute the ion flight times. We also assume that the footpoint of the field line is convecting through the ionosphere at a typical velocity, $V_{C}=1 \mathrm{~km} \cdot \mathrm{s}^{-1}$, which is roughly $8 \times 10^{-3^{\circ}}$ (in MLAT). $\mathrm{s}^{-1}$. For $\alpha<50^{\circ}$, we can see that cusp ions are predicted to be observed further equatorward at POLAR than at DMSP, due to the shorter field-aligned distance to the $X$-line. However, once $\alpha>50^{\circ}$, cusp ions are expected to be observed further poleward at POLAR than at DMSP, and $\triangle$ MLAT increases rapidly with $\alpha$. Given the differences in the ion pitch angle distributions observed at POLAR and DMSP, as discussed above, these convection effects can explain the observed $\approx 2^{\circ}$ difference in MLAT. Also, in the MICS observations there will be a contribution from upgoing ions that have mirrored at low altitudes in the cusp and are travelling in the anti-field-aligned direction away from the Earth; this would again be expected to shift the cusp observations at POLAR further poleward of those at DMSP, as observed.

In Fig. 3a, it is unlikely that there is a significant contribution from the mantle population shown in Fig. 1c of Newell and Meng, as that has a broad range in local time, unlike the occurrences shown here. Mantle ions are expected to have lower energies than cusp ions, so the majority probably fall below the energy threshold of MICS.

The distribution of average ion energies from 1.53 to $2.10 \mathrm{keV}$ is shown in Fig. 3b. The peak in the occurrence of ions at these energies is distributed between MLAT $\approx 76.5^{\circ}$ and $\approx 81.5^{\circ}$ and extends for about $8 \mathrm{~h}$ in MLT centred about 09:00 MLT. This appears to relate to the LLBL identified in Fig. 1d of Newell and Meng, which peaks between MLAT $\approx 76^{\circ}$ and $\approx 80^{\circ}$ and extends for about $6 \mathrm{~h}$ centred around 10:30 MLT. Time-of-flight arguments demand that equatorward of the cusp precipitation must lie on open LLBL (Lockwood and Smith, 1993; Lockwood, 1997). The strong pre-noon bias of these occurrences appears to also relate to the dawn asymmetry in Fig. 2b, again strongly suggesting that the majority of LLBL ions away from noon are on closed field lines and have drifted earthward from the tail. This drift can continue onto open field lines but only while the particles coming from closed field lines precipitate, mirror and return 
to the spacecraft (i.e. up to half a particle bounce period): only after this time will the effect of the lack of supply of trapped particles be seen by the spacecraft. Thus, the ions can only penetrate by drifts onto the low-latitude edge of the open polar cap. The peak in the occurrence of this LLBL ion population originating on closed field lines appears to be about $1^{\circ}$ in MLAT further poleward when observed at POLAR than when observed at DMSP. This discrepancy in MLAT could be due to the fact that the closed LLBL ions observed at DMSP have been scattered into the loss cone, whereas POLAR observes the bulk of the population on drift orbits. However, it could also reflect poleward drift, as in the case of the cusp ions discussed above, if magnetopause reconnection extends to the pre-noon sector. POLAR observes higher occurrences at around 06:00 MLT than DMSP; this is caused by low energy BPS ions which are part of the overall BPS population identified in Fig. 1e of Newell and Meng. The identification of these ions as BPS by Newell and Meng is likely due to their more sophisticated classification methods.

The open LLBL near noon observed at POLAR centred about MLAT $\approx 78.5^{\circ}$ appears to be about $0.5^{\circ}$ in MLAT further poleward of that observed by DMSP centred about MLAT $\approx 78^{\circ}$. This is a result of the convection effects used to explain the discrepancy in cusp locations discussed above. The discrepancy here is less than in the cusp $\left(\triangle \mathrm{MLAT} \approx 0.5^{\circ}\right.$ for the LLBL, as opposed to $\triangle \mathrm{MLAT} \approx 2^{\circ}$ for the cusp) due to the higher LLBL ion energies and the more field-aligned nature of LLBL population typically observed at POLAR. In both the POLAR and DMSP observations the open LLBL is equatorward of the cusp and is thus consistent with the energy-dispersed ion signatures expected to result from subsolar reconnection.

We sorted the occurrences in Figs. $3 \mathrm{a}$ and $3 \mathrm{~b}$ by the sense of the IMF $B_{Z}$ component (not shown) and showed that for both senses the cusp occurred poleward of the LLBL. These features were observed further poleward for northward IMF conditions. This is consistent with the effects of magnetopause reconnection for cusp ions, and LLBL ions drifting earthward from the tail on closed field lines (a weaker $E_{C}$ under IMF $B_{Z}>0$ causes ion drift orbits further from the Earth, which then map to higher latitudes).

Figures $3 \mathrm{a}$ and $3 \mathrm{~b}$ both have small, but significant, occurrence probabilities in the region about MLAT $\approx 90^{\circ}$. This possibly relates to polar rain, as described by Newell and Meng, though it is more likely polar wind created by upwelling ionospheric plasma (e.g. Lockwood et al., 1985).

Figure $3 \mathrm{c}$ shows the distribution for average ion energies ranging from 2.10 to $4.85 \mathrm{keV}$. In this distribution the peak occurrences range from MLAT $\approx 70^{\circ}$ to $79^{\circ}$ on the dawn flank and from MLAT $\approx 70^{\circ}$ to $81^{\circ}$ on the dusk flank. This is consistent with the BPS shown in Fig. 1e of Newell and Meng and the occurrences in Fig. 2c, both of which extend to higher latitudes at dusk. The similarity with occurrences in Fig. 2c, where the distribution is roughly symmetric about the noon-midnight meridian, indicates that the $\boldsymbol{E} \times \boldsymbol{B}$ and gradient/curvature drifts are balancing at these energies.
Figure $3 \mathrm{c}$ shows a clear gap around noon in the BPS population, consistent with these particles convecting sunward on closed field lines. However, there are a significant number of cases in which $>2 \mathrm{keV}$ ions are seen within this gap. From their MLAT and MLT, these lie immediately equatorward of the open LLBL and cusp particles near noon and thus are likely to be open BPS particles as proposed by Lockwood (1997). These could have resulted from acceleration of magnetospheric particles at the interior RD launched by the reconnection site (standing in the inflow to the magnetopause on the magnetospheric side of the boundary, whereas the main magnetopause current sheet is the corresponding RD standing in the inflow on the magnetosheath side), as modelled by Lockwood et al. (1996). Alternatively, these may have resulted from acceleration at the bow shock, as proposed by Fuselier et al. (1999). Such "open BPS" is likely to have very narrow latitudinal width and so could have been missed in this survey with $1.5^{\circ}$ MLAT bin widths. However, occurrences were only slightly increased in the survey that used $0.5^{\circ}$ MLAT bin width. We conclude that some BPS particles can be found on open field lines, but they are rare - possibly because the interior RD and/or the Fermi acceleration mechanisms only rarely elevate ions to BPS energies and most such particles are found in the open LLBL.

Figure $3 \mathrm{~d}$ shows the distribution for energies ranging from 4.85 to $8.55 \mathrm{keV}$. The peaks in the occurrences are centred about MLAT $\approx 70^{\circ}$ on both flanks, and cover a broader range of latitudes on the dawn flank than on the dusk flank. By comparison with occurrences in Figs. 1e and 1f of Newell and Meng, the dawn flank occurrences relate to the CPS and the dusk flank occurrences relate to the BPS, respectively. We also notice the appearance of occurrences below MLAT $\approx 67^{\circ}$ in Figs. $3 \mathrm{c}$ and d, particularly on the dawn flank.

Figures $3 \mathrm{e}$ and $\mathrm{f}$ show the distribution of ions with energies from 8.55 to $15.20 \mathrm{keV}$ and 15.20 to $26.83 \mathrm{keV}$, respectively. The narrow band of occurrences on the dawn flank centred about MLAT $\approx 65^{\circ}$ in Fig. $3 \mathrm{e}$ is consistent with the CPS shown in Fig. 1f of Newell and Meng. However, the peak in the occurrences are beginning to slip below MLAT $\approx 67^{\circ}$ (at these latitudes MLAT $\approx \Lambda$, so $L \approx 6.6$ ), which means that ring current ions are beginning to contribute to the background occurrences. These ions are trapped on closed field lines and have not immediately drifted earthward from the tail. We also note that these occurrences tend toward the dusk flank, showing a change in asymmetry at these latitudes compared with the occurrences shown in Figs. 3c and 3d. Again, this fits with the drift orbit hypothesis for ions at these energies drifting around to the dayside magnetopause from the dusk flank and scattering at the magnetopause before reaching the dawn flank (Lyons and Williams, 1984b). The portion of the distribution in Figs. $3 \mathrm{c}$ and $3 \mathrm{f}$ below MLAT $\approx 65^{\circ}$ at dawn and MLAT $\approx 70^{\circ}$ at dusk appears to relate to the region described by Newell and Meng as void where there is negligible ion precipitation into the ionosphere.

Within the BPS/CPS precipitation regions there is the transition from $\boldsymbol{E} \times \boldsymbol{B}$ to gradient/curvature drift dominated flow. This transition is expected to be a gradual process from $\mathrm{keV}$ 
to tens of $\mathrm{keV}$ energies, as ion trajectories evolve from flowing mostly towards dawn to flowing mostly toward dusk (see Figs. 4.25 and 4.27 of Lyons and Williams, 1984b). The narrow band of high occurrences in Fig. 3e marks the full transition to gradient/curvature drift dominated ion trajectories. This qualitatively explains the distribution of ions precipitating into the auroral oval.

\section{Discussion and conclusions}

The surveys presented in this paper enable us to relate the studies of Stubbs et al. and Newell and Meng in terms of both ion energy and location in latitude and MLT. We are able to qualitatively explain the lower DCR cusp energies relative to the $\mathrm{He}^{2+}$ ion survey by magnetic reconnection related acceleration processes at the magnetopause. We show that the similar decrease in the magnitude of the cross-over energy for particles on drift orbits can be explained in terms of the competing $\boldsymbol{E} \times \boldsymbol{B}$ and gradient/curvature drifts, and acceleration by Alfvén waves as ions cross the tail current sheet (Onsager and Mukai, 1995). The ion observations here also support the observations and interpretations of Friedel et al. (2001), based mainly on electron data, that plasma transport can be well explained by drift orbit theory.

Open field line features are observed at energies around $\approx 1 \mathrm{keV}$ in the DCR channel and distributed about noon with a slight pre-noon bias. As expected, ion occurrences are observed at lower latitudes in Fig. 3 than in Fig. 2 due to the differences between $\Lambda$ and MLAT discussed in Sect. 2. There are also slight differences between the energies observed in Figs. 2 and 3, due to the differences in determining the peak in the $J_{E}$ spectra and calculating the average ion energy, as detailed in Sect. 3.

Cusp and open LLBL ions observed at POLAR, as shown in Figs. 3a and b, respectively, are around $\approx 1^{\circ}$ to $2^{\circ}$ higher in MLAT than those observed by Newell and Meng. However, these differences are shown to be consistent with the differences expected due to energy and pitch-angle dependent dispersion caused by convection during the time-offlight from the reconnection $X$-line to POLAR or DMSP. These effects mean that the slower an ion's field-aligned velocity, the longer it remains on a field line; thus allowing it to reach higher latitudes the further it convects in the antisunward direction. The DMSP SSJ/4 instruments only observe near field-aligned ions (which have the shortest timeof-flight from the reconnection $X$-line) compared with MICS aboard POLAR, which observes ions with a range of pitch angles that have relatively longer time-of-flights. Hence, even though POLAR was closer to the $X$-line, it observes ions at higher latitudes than DMSP, as the field-aligned velocities of the ions observed by MICS were less than those observed by DMSP for any given energy. In Fig. 4 we show that we expect the cusp to be observed further poleward by POLAR for ions with pitch angles greater than $\approx 50^{\circ}$. The poleward location of the cusp at POLAR could also be in part due to ions that have mirrored at low altitudes and are travelling away from the Earth. We also note that the open LLBL around noon is, on average, equatorward of the cusp, so this is consistent with the energy-dispersed signature expected for subsolar reconnection.

We also present evidence in Figs. $2 b$ and $3 b$ that the prenoon bias in the occurrence of LLBL particles observed by Newell and Meng is most likely caused by the component of the LLBL coming from closed field lines formed by low energy ions drifting earthward from the tail; and thus relates in part to the dawn-dusk asymmetry observed by Stubbs et al. in $\mathrm{He}^{2+}$ ion occurrences. These ions mainly remain on closed field lines but the drift can continue onto open field lines for up to half a particle bounce period. We also note that the occurrences around 06:00 MLT in Fig. 3b have a contribution from the lower energy component of the BPS ion population, as identified in Fig. 1e of Newell and Meng. The implications for the formation of the LLBL and momentum transfer are important. There is an open LLBL immediately equatorward of the cusp caused by reconnection and there appears to be an LLBL on the dawn flank only, consistent with particles drifting sunward. These ions can penetrate onto open field lines but only in a thin latitudinal band and will primarily be on closed field lines. Thus, the sheath plasma on the open LLBL field lines is consistent with entry along open field lines that subsequently close in the tail and convect sunward. We conclude that the closed LLBL does not provide evidence for transfer of mass and momentum from the solar wind to the LLBL by some non-reconnection ("viscous-like") interaction, but that these ions were originally injected onto open field lines which were subsequently closed by tail reconnection. The observed LLBL ions then migrated sunward from the tail, preferentially to the dawn flank because their energy was below the "cross-over energy".

At higher energies $(\approx 2$ to $20 \mathrm{keV})$, the distribution tends towards the region of the auroral oval. This relates to the BPS and CPS populations, where ions make the transition from dawnward to duskward dominated flow with increasing energy to form the dawn-dusk asymmetry. In particular, the ion population at the cross-over energy seems to relate to the BPS in terms of both energy and location in latitude and MLT. Table 1 shows that both the BPS and CPS ions have higher energies at dusk than at dawn. This leads to the occurrences from the dawn BPS appearing in Figs. $3 b$ and $c$ and the dusk BPS appearing in Figs. $3 \mathrm{c}$ and d. This is similarly shown for the CPS in Figs. 3d and e. These overlaps in the average energy and location of the plasma populations classified by Newell and Meng are to be expected, as they used a more sophisticated set of selection criteria.

We have related statistical observations made at different altitudes in the magnetosphere-ionosphere system and shown them to be consistent. We have explained the observed occurrence distributions in terms of ion injection at the dayside magnetopause and the transport of ions earthward from the tail along drift orbit trajectories. We have also produced evidence that the component of the LLBL on closed field lines has drifted earthward from the tail and, therefore, has not entered directly from the dayside magnetopause. This has 
important consequences for how we understand the coupling between the solar wind and magnetosphere. Ions at energies around the cross-over energy relate to the BPS and CPS populations, and are thus important in understanding the influence of drift orbits on the location and behaviour of ions in the auroral oval. To fully test the qualitative explanations given here for these observations will require a comparison with numerical simulations; this will hopefully be the subject of a future paper.

\section{Appendix A \\ Deriving the estimate for the cross-over energy}

We calculate a crude estimate for the cross-over energy to establish if the flip in asymmetry about the noon-midnight meridian can be explained by drift orbits. This estimate is only expected to be approximately accurate, as we need to make simplifying assumptions to obtain an analytical solution. The drifts due to the electric field $(\boldsymbol{E})$ and the gradient $(\nabla B)$ and curvature $\left(R_{C}\right)$ of the magnetic field are given by Eqs. (A1), (A2) and (A3), respectively.

$$
\begin{aligned}
& \boldsymbol{V}_{E}=\frac{\boldsymbol{E} \times \boldsymbol{B}}{B^{2}} \\
& \boldsymbol{V}_{\nabla}=\frac{m v_{\perp}^{2}}{2 q B^{3}}(\boldsymbol{B} \times \nabla B) \\
& \boldsymbol{V}_{R}=\frac{m v_{\|}^{2}}{q} \frac{\boldsymbol{R}_{c} \times \boldsymbol{B}}{R_{C}^{2} B^{2}}
\end{aligned}
$$

In the above equations: $v_{\perp}$ and $v_{\|}$are the perpendicular and parallel velocity components relative to the magnetic field $(\boldsymbol{B}) ; m$ and $q$ are particle mass and charge, respectively. If we assume that currents are negligible, we can combine the gradient and curvature drifts, $\boldsymbol{v}_{R}$ and $\boldsymbol{v}_{\nabla}$, respectively, to obtain the total magnetic drift,

$$
\begin{gathered}
\boldsymbol{V}_{B}=\boldsymbol{V}_{R}+\boldsymbol{V}_{\nabla}=\left(\boldsymbol{v}_{\|}^{2}+\frac{1}{2} v_{\perp}^{2}\right) \frac{\boldsymbol{B} \times \nabla B}{\omega_{g} B^{2}}= \\
\frac{W}{q B^{3}}\left(1+\cos ^{2} \alpha\right) \boldsymbol{B} \times \nabla B ;
\end{gathered}
$$

where $\omega_{g}$ is the particle gyro-frequency (Lyons and Williams, 1984a; Baumjohann and Treumann, 1997). In reality, a particle in a dipole field will gyrate, bounce and drift all at the same time. To determine the slower drift motion we must integrate over the gyration and bounce motions. The average total magnetic drift is given by

$<v_{B}>\approx \frac{6 W}{q B_{e q} r_{e q}}\left(0.35+0.15 \sin \alpha_{e q}\right)$,

where $r_{e q}$ is the equatorial crossing distance from the centre of the Earth, and $B_{e q}$ is the magnetic field magnitude in the equatorial plane (See Lyons and Williams (1984a) or Stubbs (2002) for a more rigorous derivation).
As can be seen from Eq. (A5), $\left\langle v_{B}>\right.$ has only a weak dependence on equatorial pitch angle. There is a stronger dependence on particle charge, energy and magnetic field geometry, but no dependence on particle mass (Lyons and Williams, 1984a). We would thus expect different ion species with the same energy and charge state to drift with the same magnetic drift velocity, e.g. $\mathrm{H}^{+}$and $\mathrm{He}^{+}$ions.

The solar wind generates an electric field inside the magnetosphere, $\boldsymbol{E}=-\boldsymbol{V}_{s w} \times \boldsymbol{B}$, which is able to penetrate via open magnetic flux, particularly during periods of southward IMF. This electric field is referred to as the convection electric field, $E_{C}$, and is directed from dawn to dusk in the equatorial plane, so particles will experience an $\boldsymbol{E} \times \boldsymbol{B}$ drift, as described by Eq. (A1).

We can obtain an average value for the electric field drift velocity,

$<v_{E}>\approx \frac{E_{\perp e q}}{B_{e q}}$

(Lyons and Williams, 1984a; Stubbs, 2002). Here $E_{\perp e q}$ is the electric field perpendicular to the Sun-Earth line in the equatorial plane.

We can write Eq. (A5) for the average total magnetic drift velocity and Eq. (A6) for the average electric drift velocity as a function of the magnetic shell, $L$ (assumed to be equivalent to the McIlwain $L$-shell) to give

$<v_{B}>\approx \frac{6 W L^{2}}{q B_{E} R_{E}}\left(0.35+0.15 \sin \alpha_{e q}\right)$

and

$<v_{E}>\approx \frac{E_{\perp e q} L^{3}}{B_{E}}$.

In these equations, $B_{E}$ is the equatorial magnetic field magnitude at the surface of the Earth, $R_{E}$ is the radius of the Earth, and $\alpha_{e q}$ is the particle pitch angle in the equatorial plane. (Here we have also used the equations: $r_{e q}=L R_{E}$ and $B_{e q}=B_{E} / L^{3}$.)

If we assume that the cross-over energy, $K_{c e}$, occurs when the drift velocities are equal and opposite, then from Eq. (A7) and (A8) we obtain

$K_{c e}=\frac{q E_{\perp e q} L R_{E}}{6\left(0.35+0.15 \sin \alpha_{e q}\right)}$.

Given the assumption that the pitch angle distribution at the equator is isotropic, we can substitute $\alpha_{I}$ for $\alpha_{e q}$, where $\sin \alpha_{I}=\sqrt{\left(\frac{2}{3}\right)}$, given that $v_{\perp}^{2}=2 v_{\|}^{2}$ for an isotropic distribution. We also assume that the dominant electric field in the near-Earth tail is the convection electric field, $E_{C}$. Using these substitutions we obtain

$K_{c e} \approx G Q E_{C} L=2.3 Q E_{C} L$.

Here $K_{c e}$ is the cross-over energy $(\mathrm{keV}) ; Q$ is the charge state (dimensionless integer); $E_{C}$ is the convection electric 
field (mV.m $\mathrm{m}^{-1}$ ); $L$ is the $L$-shell (magnetic shell, dimensionless); $G$ is a constant $\left(\mathrm{keV} .\left(\mathrm{mV} . \mathrm{m}^{-1}\right)^{-1} \cdot R_{E}^{-1}\right)$.

We assume that an isotropic equatorial ion pitch angle is reasonable as $K_{c e}$ does not have a strong $\alpha_{e q}$ dependence. This can be demonstrated by calculating $G$ for an $\alpha_{e q}$ of $10^{\circ}$ and $90^{\circ}$, which gives values of $\approx 2.8$ and $\approx 2.1$, respectively.

Acknowledgements. This work was funded by PPARC. T. J. Stubbs was also funded by the National Research Council whilst working at NASA/GSFC. We thank the many individuals who have made the CAMMICE program (PI T. A. Fritz) a success. We are grateful to P. T. Newell for useful discussions on how best to compare our results with his DMSP study. Thanks to S. J. Schwartz for discussions about ions in the magnetosheath. Thanks also to J. Scuffham for assistance with the T01 model. We benefited from use of WIND MFI and SWE data (PIs R. P. Lepping and K. W. Ogilvie, respectively) obtained from CDAWeb and Dst data obtained from NSSDC OMNIWeb, both of which are managed by SPDF at NASA/GSFC. Figure 1 was reproduced and modified by permission of the American Geophysical Union.

Topical Editor T. Pulkkinen thanks two referees for their help in evaluating this paper.

\section{References}

Acuña, M. H., Ogilvie, K. W., Baker, D. N., Curtis, S. A., Fairfield, D. H., and Mish, W. H.: The global geospace science program and its investigations, Space Sci. Rev., 71, 5-21, 1995.

Baker, K. B. and Wing, S.: A new magnetic coordinate system for conjugate studies at high-latitudes, J. Geophys. Res., 94, 91399143, 1989.

Baumjohann, W. and Treumann, R.A.: Basic Space Plasma Physics, Imperial College Press, 1997.

Eastman, T. E., Popielawska, B., and L. A. Frank: Threedimensional plasma observations near the outer magnetospheric boundary, J. Geophys. Res., 90, 9519-9539, 1985.

Feldstein, Y. I. and Galperin, Yu.I.: The auroral luminosity structure in the high-latitude upper atmosphere: Its dynamics and relationship to the large-scale structure of the Earth's magnetosphere, Rev. Geophys., 23, 217-275, 1985.

Friedel, R. H. W., Korth, H., Henderson, M. G., Thomsen, M. F., and Scudder, J. D.: Plasma sheet access to the inner magnetosphere, J. Geophys. Res., 106, A4, 5845-5858, 2001.

Fritz, T .A., Daglis, I. A., Livi, S., Sommer, H., Wilken, B., Grande, M., Hall, D. D., Blake, J.B. Fennell, J. F., and Koga, R.: The POLAR CAMMICE investigation, Tech. Rep., Centre for Space Physics, Boston, 1997.

Fuselier, S. A., Klumpar, D. M., Shelley, E. G., Anderson, B. J., and Coates, A. J.: $\mathrm{He}^{2+}$ and $\mathrm{H}^{+}$dynamics in the subsolar magnetosheath and plasma depletion layer, J. Geophys. Res., 96, A12, 21 095-21 104, 1991.

Fuselier, S. A., Lockwood, M., Onsager, T. G., and Peterson, W. K.: The source population for the cusp and cleft/LLBL for southward IMF, Geophys. Res. Lett., 26, 1665-1669, 1999.

Galperin, Yu. I. and Feldstein, Y. I.: Auroral luminosity and its relationship to magnetospheric plasma domains, in: Auroral Physics, edited by Meng, C.-I., Rycroft, M. J., and Frank, L. A., Cambridge University Press, New York, 1991.

Ghielmetti, A. G., Johnson, R. G., Sharp, R. D., and Shelley, E. G.: The latitudinal, diurnal, and altitudinal distributions of upward flowing energetic ions of ionospheric origin, Geophys. Res. Lett., 5, 59-62, 1978.

Hardy, D. A., Yeh, H. C., Schmitt, L. K. Schumaker, T. L., Gussenhoven, M. S., Huber, A., Marshall, F. J., and Pantazis, J.: Precipitating electron and ion detectors ( $\mathrm{SSJ} / 4$ ) on the block 5D/Flights 6-10 DMSP satellites: Calibration and data presentation, Tech. Rep. AFGL-TR-84- 0317, Air Force Geophys. Lab., Hanscom Air Force Base, Mass., 1984.

Hudson, P. D.: Discontinuities in an anisotropic plasma and their identification in the solar wind, Planet. Space Sci., 18, 1611$1622,1970$.

Hultqvist, B., Lundin, R., Stasiewicz, K., Block, L., Lundqvist, P.-A., Gustafson, G., Kaskinen, H., Bahnsen, A., Potemra, T. A., and Zanetti, L. J.: Simultaneous observations of upward moving field-aligned electrons and ions on auroral zone field lines, J. Geophys. Res., 93, 9765-9776, 1988.

Lockwood, M., Waite Jr., J. H., Moore, T. E., Johnson, J. F. E., and Chappell, C. R.: A new source of suprathermal $\mathrm{O}^{+}$near the dayside polar cap boundary, J. Geophys. Res., 90, 4099-4116, 1985.

Lockwood, M. and Smith, M. F.: Comment on "Mapping the dayside ionosphere to the magnetosphere according to particle precipitation characteristics" by Newell and Meng, Geophys. Res. Lett., 20, 1739-1740, 1993.

Lockwood, M., Cowley, S. W. H., and Onsager, T. G.: Ion acceleration at both the interior and exterior Alfvén waves associated with the magnetopause reconnection site: signatures in cusp precipitation, J. Geophys. Res., 101, 21 501-21 515, 1996.

Lockwood, M.: Relationship of dayside auroral precipitations to the open-closed separatrix and the pattern of convective flow, J. Geophys. Res., 102, A8, 17 475-17487, 1997.

Lyons, L. R. and Williams, D. J.: Chapter 2: Charged particle motion in magnetic and electric fields, Quantitative aspects of magnetospheric physics, D. Reidel Publishing Co., 1984a.

Lyons, L. R. and Williams, D. J.: Chapter 4: Electric fields, Quantitative aspects of magnetospheric physics, D. Reidel Publishing Co., 1984b.

McIlwain, C. E.: Magnetic coordinates, Space Sci. Rev., 5, 585598, 1966.

Mizera, P. F. and Fennell, J. F.: Signatures of electric fields from high and low altitude particle distributions, Geophys. Res. Lett., 4, 311-314, 1977.

Newell, P. T. and Meng, C.-I: The cusp and the cleft/LLBL: Low altitude identification and statistical local time variation, J. Geophys. Res., 93, 14 549-14 556, 1988.

Newell, P. T. and Meng, C.-I.: Intense keV energy polar rain, J. Geophys. Res., 95, 7869-7879, 1990.

Newell, P. T., Burke, W. J., Meng, C.-I., Sanchez, E. R., and Greenspan, M. E.: Identification and observations of the plasma mantle at low altitude, J. Geophys. Res., 96, 35-45, 1991a.

Newell, P. T., Wing, S., Meng, C.-I., and Sigillito, V.: The auroral oval position, structure, and intensity of precipitation from 1984 onward: An automated on-line data base, J. Geophys. Res., 96, 5877-5882, 1991b.

Newell, P. T., Burke, W. J., Sànchez, E. R., Meng, C.-I.: Greenspan, M. E., and Clauer, C. R.: The low-latitude boundary layer and the boundary plasma sheet at low altitude: Prenoon precipitation regions and convection reversal boundaries, J. Geophys. Res., 96, 21 013-21 023, 1991c.

Newell, P. T. and Meng, C.-I: Mapping the dayside ionosphere to the magnetosphere according to particle precipitation characteristics, Geophys. Res. Lett., 19, 609-612, 1992. 
Onsager, T. G. and Mukai, T.: Low altitude signature of the plasma sheet boundary layer: Observations and model, Geophys. Res. Lett., 22, 7, 855-858, 1995.

Phan, T.-D., Paschmann, G., Baumjohann, W., Sckopke, N., and Lühr, H.: The magnetosheath region adjacent to the dayside magnetopause: AMPTE/IRM observations, J. Geophys. Res., 99, A1, 121-141, 1994.

Phan, T.-D., Paschmann, G., and Sonnerup, B. U. Ö.: Low-latitude dayside magnetopause and boundary layer for high magnetic shear, 2. Occurrence of magnetic reconnection, J. Geophys. Res., 101, A4, 7817-7828, 1996.

Sckopke, N., Paschmann, G., Haerendel, G., Sonnerup, B. U. Ö., Bame, S. J., Forbes, T. G., Hones, E. W., and Russell, C. T.: Structure of the low-latitude boundary layer, J. Geophys. Res., 86, 2099-2110, 1981.

Scudder, J., F. Hunsacker, G. Miller, J. Lobell, T. Zawistowski, K. Ogilvie, J. Keller, D. Chornay, F. Herrero, R. Fitzenreiter, D. Fairfield, J. Needell, D. Bodet, J. Googins, C. Kletzing, R. Torbert, J. Vandiver, R. Bentley, W. Fillius, C. McIlwain, Whipple, E., and A. Korth, HYDRA A 3-dimensional electron and ion hot plasma instrument for the POLAR spacecraft of the GGS mission, Space Sci. Rev., 71, 459-495, 1995.

Shelley, E.G.: Magnetospheric energetic ions from the Earth's ionosphere, Adv. Space Res., 6, 121-132, 1986.

Stubbs, T. J., Cargill, P., Grande, M., Kellett, B., Lockwood, M., and Perry, C.: Observations of the northern polar cusp with the POLAR spacecraft, Proc. Cluster-II Workshop on Multiscale/Multipoint Plasma Measurements, London, 22-24 September 1999, ESA SP-449, February 2000.

Stubbs, T. J., Lockwood, M., Cargill, P., Fennell, J., Grande, M., Kellett, B., Perry, C., and Rees, A.: Dawn-dusk asymmetry in particles of solar wind origin within the magnetosphere, Ann. Geophys., 19, 1-9, 2001.
Stubbs, T. J.: Ion injection and transport in the high-latitude magnetosphere, Ph. D. Thesis, Imperial College, London, 2002.

Stubbs, T. J., Lockwood, M., Cargill, P. J., Grande, M., Kellett, B., and Perry, C.: Extended cusp- like regions and their dependence on interplanetary conditions, submitted to J. Geophys. Res., July 2003.

Tsyganenko, N. A.: A model of the near magnetosphere with a dawn-dusk asymmetry 1. Mathematical structure, J. Geophys. Res., 107, A8, art. no. 1179, 2002a.

Tsyganenko, N. A.: A model of the near magnetosphere with a dawn-dusk asymmetry 2. Parameterization and fitting to observations, J. Geophys. Res., 107, A8, art. no. 1176, 2002 b.

Wilken, B., Wei, W., Hall, D., Grande, M., Søraas, F., and Fennell, J. F.: Magnetospheric ion composition spectrometer onboard the CRRES spacecraft, J. Spac. Rock., 29, 585-591, 1992.

Whipple, E. C.: (U, B, K) coordinates: A natural system for studying magnetospheric convection, J. Geophys. Res., 83, 43184326, 1978.

Winningham, J. D. and Heikkila, W. J.: Polar cap auroral electron fluxes observed with ISIS-1, J. Geophys. Res., 79, 949-957, 1974.

Winningham, J. D., Yasuhara, F., Akasofu, S.-I., and Heikkila, W. J.: The latitudinal morphology of $10-\mathrm{eV}$ to $10-\mathrm{keV}$ electron fluxes during magnetically quiet and disturbed times at the 21:0003:00 MLT sector, J. Geophys. Res., 80, 3148, 1975.

Wolf, R. A.: Magnetospheric configuration, Introduction the Space Physics, edited by Kivelson, M. G. and Russell, C. T., Cambridge University Press, 1995. 